\title{
BER analysis of concatenated levels of encoding in GFDM system using labview
}

\author{
Nagarjuna Telagam ${ }^{1}$, S. Lakshmi ${ }^{2}$, K. Nehru ${ }^{3}$ \\ ${ }^{1,2}$ Department of Electronics and Communication Engineering, Sathyabama Institute of Science and Technology, India \\ ${ }^{3}$ Department of Electronics and Communication Engineering, Institute of Aeronautical Engineering, India
}

\begin{tabular}{l}
\hline \hline Article Info \\
\hline Article history: \\
Received Sep 10, 2018 \\
Revised Nov 27, 2018 \\
Accepted Jan 11, 2019
\end{tabular}

\section{Keywords:}

BER

Channel Coding

GFDM

Labview

SNR

VI

\begin{abstract}
All the devices are interconnected each other in digital form, for different applications the input data is encoded for error correcting and detecting purpose. The paper describes the transmission of QAM signals with two level encoded stages, i.e. convolutional and hamming coded GFDM system with 256-point IFFT at transmitter and FFT at the receiver using LABVIEW software. GFDM is a non-orthogonal, digital multicarrier transmission scheme which digitally implements the classical filter bank approach. GFDM transmits a block of frame composed by M time slots with K subcarriers. The higher order QAM is used because of transmitting more data but is less reliable when compared to lower order QAM. Based on GFDM specifications for the IEEE 802.11, latest 5G physical layer standards, the coding is provided by $1 / 2$ rate encoder at the input side, and Maximum Likelihood decoder at the receiver side is used. The standard convolution code $(7,[171,133])$, is used as encoder for the GFDM system. The GFDM complex values are displayed in the front panel, along with FFT and power spectrum is plotted for GFDM signal. The array of input bits and output bits are shown with green colour LED's. The van de Beek algorithm is used at the receiver for maximum likelihood detection acts as convolutional decoder of GFDM signal. Next the signal is subjected to remove cyclic prefix and zero padding and applied to channel estimation algorithm. The un-equalized data and equalized data graph is shown in the front panel, before and after channel estimation VI. With BER VI available in the LABVIEW the data is normalized and its response is plotted with respect to SNR. BER values for different levels of encoders have shown in table for SNR values. This paper concludes the $32.91 \%$ improvement in BER for two levels of concatenated codes. Thus the GFDM signal outperforms the OFDM signal interms of BER for series levels of coding using labVIEW software.
\end{abstract}

Copyright () 2019 Institute of Advanced Engineering and Science. All rights reserved.

\section{Corresponding Author:}

NagarjunaTelagam,

Research Scholar, School of Electronics,

Department of Electronics and Communication Engineering,

Sathyabama Institute of Science and Technology,

Campus Road, Rajiv Gandhi Salai, Jeppiaar Nagar, Chennai, Tamil Nadu 600119.

Email: nagarjuna473@gmail.com

\section{INTRODUCTION}

Long term evolution (LTE) being deployed worldwide and research on $5^{\text {th }}$ Generation is under way. The major requirement is short latency (less than $1 \mathrm{~ms}$ ) and high energy efficiency. Dynamic spectrum access is the dominant technology in the coming generation for high power spectral density. The authors of the paper considered FBMC, GFDM as waveforms and designed cognitive radio to use fragmented spectrum and it provides high bandwidth efficiency and better subcarrier separation [1]. Reclined synchronization between 
the mobile users in $5 \mathrm{G}$ is needed. OFDM signal suffers from carrier frequency offset because OFDM symbols expand in the frequency domain. In addition to that, the Out of Band radiation for OFDM is $-35 \mathrm{~dB}$ which is not suitable for 5G Requirements [2]. OFDM signal is not preferred because of its drawbacks, especially PAPR Problem. Although many algorithms have proposed and evaluated this problem but still it is not considered for 5G. The candidate waveform GFDM is slightly suffers from PAPR problem because of the power amplifiers in the transceiver, and they will saturate and cause reduction in BER and OOB. The authors evaluate this problem by proposing the clipping techniques with iterative receiver companding and mapping techniques which avoids the PAPR and observed the improvement in BER [3]. There is a letter which proposes GFDM system performance can be increase by applying the Balian law theorem which proves the nonexistence of ZF receivers [4]. In 5G the machine two machine communication needs synchronization and less BER. With the lack of synchronization between the users results in carrier frequency offsets and residual symbol time.

This paper proposes the scheme to predict the BER under synchronization errors andevaluates the performance loss introduced by the synchronization [5]. GFDM which makes more widespread of timefrequency mapping of modulated signals and acquires the cyclic prefix block signalling structure from OFDM. The authors in this paper identified the synchronization problem from non-orthogonal pulse shaping causes interference among the symbols [6].The major applications in 5G Cellular networks has many challenges from a number of different scenarios such as IoT, MTC, WRAN and enhanced broad band. All these applications require better frequency efficiency. The GFDM spectrum was tested on USRP device in LABVIEW software the signal on each subcarrier is shaped by a pulse shaping filter with roll off factor values [7]. Another challenging application for the fifth generation is tactile internet. For this application the authors integrated GFDM with Walsh Hadamard transform. This assimilation is convenient for low latency applications for fifth generation [8]. Currently LTE licensed is accompanied by 4G users and the upcoming different radio access technologies will co-exist and cause interference. The authors explained the scenario for upcoming 5G applications and mentioned 5G users require backhaul signalling provided by widelydeployed LTE infrastructure.

Multicarrier modulation supports these mobile applications because of its advantages such as equalization techniques, adaptive modulation and coding [9]. The codes which are also used as inner codes with burst error correcting capability to form concatenated codes [10]. OFDM is coded with convolutional encoder to fight against frequency selective channels, this article compares BER of partialdifferential space time OFDM with coherent OFDM [11]. Hamming code is one of convolutional encoder, if it satisfies three conditions such as number of check bits should be always greater than three, and the block length is equal to $2 q-1$, final one condition is the minimum distance between the codes is 3 . These codes are used in digital subscriber lines [12]. In this article, a novel half and half space-time square coding (HSTBC) conspire is proposed for moderating sign blurring and also improving the execution of MIMO-OFDM based WLANs. Reproduction results demonstrate that the HSTBC plot extensively diminished the impact of multipath blurring in remote correspondence frameworks with enhanced bit error rate [13]. In this paper, hybrid concatenated super-orthogonal space-time trellis codes (SOSTTC) applying iterative translating are proposed for level fading channels. The proposed topologies are appeared to perform superior to anything existing connected plans with aconstituent code of convolutional and space-time codes in writing [14]. This paper explains the concept of correlation among the carrier signals and sub channels.

The spectral efficiency was calculated for the OFDM signals. In sum, correlation among the subchannels causes the degradation in the spectral efficiency of MIMO channels [15]. Different recieving antenna wire arrangements can be utilized to expand the information throughput decreasing the impacts of multipath blurring and obstruction when channel data transfer capacity is restricted. Symmetrical Space Time Block Codes alongside Transmit reception apparatus determination can enhance the execution of numerous info different yield frameworks [16]. There are some recent works on wavelet based OFDM system which has drawback in out of band radiation. Next work is the investigation of using turbo codes at the channel coding is used to improve the performance of DWT-OFDM System over Selective Fading Channel. This system provides lack of synchronization at the receiver section. The channel coded hamming code is used for 64 bit single error detection and correction using VHDLand Transmission Gate Logic is designed using OFDM. This system provides perfect synchronization along with degradable BER values for different SNR values. This work implements the convolution encoder and Viterbi decoder for constraint length 7 and Bit Rate $1 / 2[17]$. This paper codes the GFDM signal digital data with hamming and convolutional encoder in a series concatenated encoder/decoder levels. The labVIEW software is used. The advanced signal processing tools are used to develop the VI code. The schematic structure for both transmitter and receiver is designed using complex labVIEW code. The purpose of doing this experiment was to gain experience in RF communication signal processing tools and to compare the bit error rate of conventional system i.e. OFDM signal with respect to SNR values. The results shows the non orthogonal multiple access technique provides 
better simulation result compare to the existing system. The two levels of encoding of are used to code the NOMA (Non orthogonal Multiple access) GFDM signal. The purpose of this paper is to compare the simulation results of GFDM with OFDM signal.

\section{SYSTEM MODEL}

Figure 1 shows the basic block diagram of concenated levels of encoding in GFDM system. The data source is just PN sequence which generates random bits, the first level of encoding stage is convolutional encoder and followed by series level of encoding with hamming encoder. The QAM is used as constellation mapper and demapper at both transmitter and receiver. The GFDM modulator internal structure is explained in Figure 2. The corresponding VI's used in the receiver are VandeBeek algorithm is act as decoder for the series level of encoding in the transmitter. The channel estimation VI is Estimates channel based on received reference symbols and known reference symbols. Finally the data bits are received at the output.

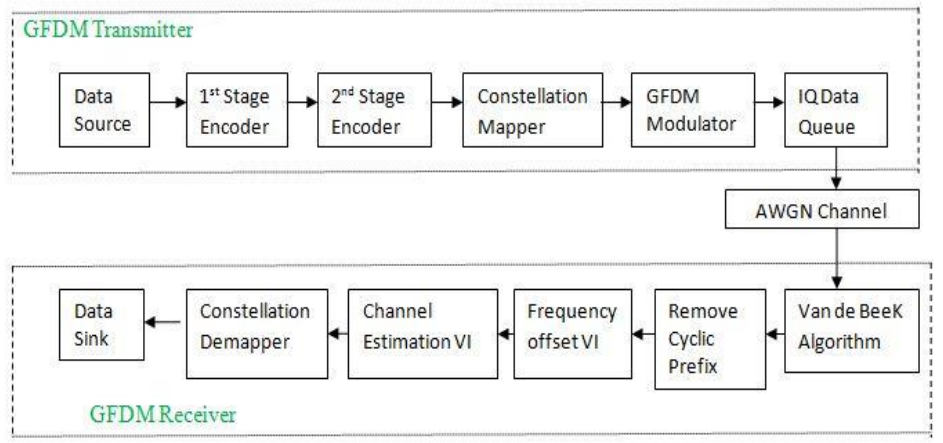

Figure 1. Block diagram of GFDM using Virtual instrumentation program

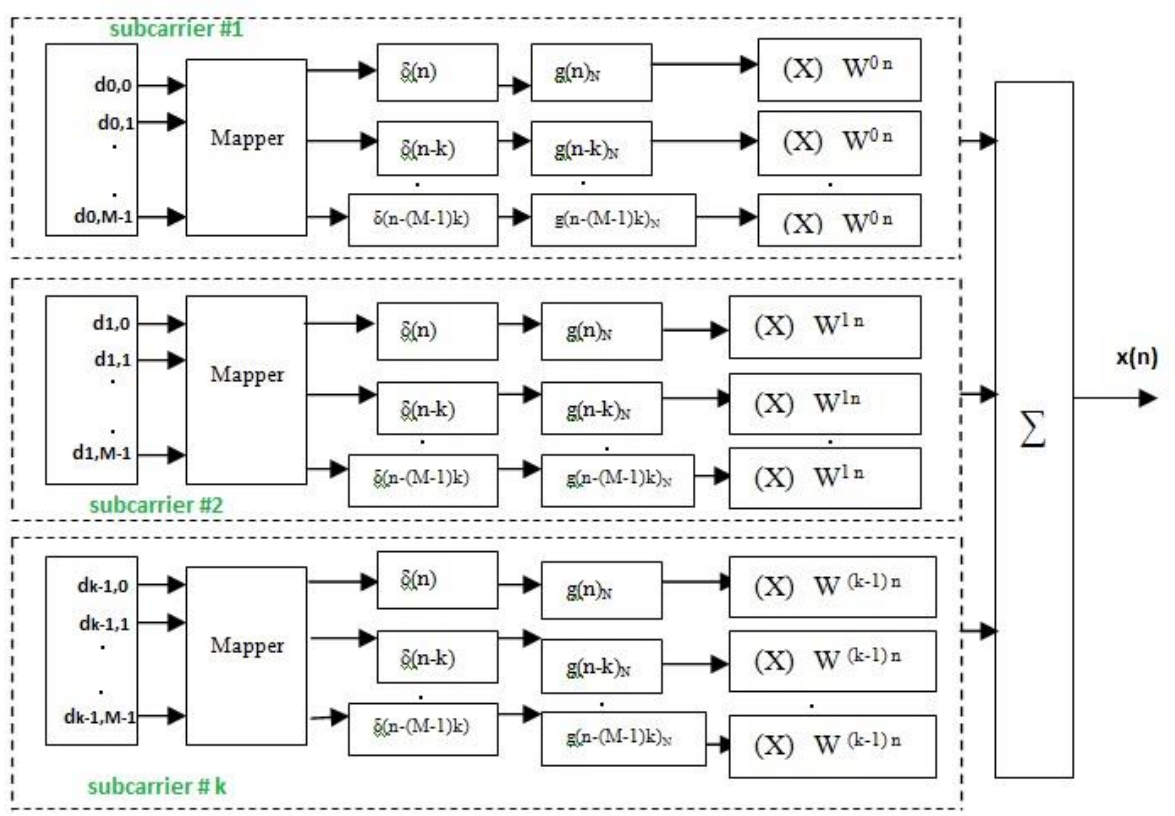

Figure 2. GFDM Modulator block diagram

Figure 2 shows the working operation of GFDM system. The data symbols is transmitted in pulse format, each symbol is time shifted version of the filter and circular modulo $\mathrm{N}$ operation is performed to rotate the pulses in $\mathrm{H}$ matrix and resulting complex values are added to generate the GFDM signal. 
The time domain and frequency domain orthogonality are two important aspects in OFDM, if we go to frequency domain the Fourier transform of pulse is sinc function, so there will be lot of side lobes, and if there is any problem in terms of synchronization, suppose if sampling is applied then it results in leakage energy, this leads to ICI, mostly occur in Wide band dispersive channel, where the both time dispersion and time variation takes place, time variation leads to Doppler shift, there is guard band between each and every symbol this is again loss of spectral efficiency, The main advantage in OFDM is Equalization because of $\mathrm{H}$ matrix and it can be decomposed into Eigen matrix, the PAPR is very high in OFDM, the two alternatives are there to overcome this OFDM are GFDM, OQAM. GFDM has many advantages here the pulse is no more rectangle, pulse duration in OFDM is from 0 to $\mathrm{T}$, but in GFDM the pulse duration is not from 0 to $\mathrm{T}$. The long duration pulse is used in GFDM, so the long pulse is used which makes the frequency domain is flat. The combination of subcarriers and sub symbol make one symbol in GFDM, the time versus frequency grid is required to analyze the subcarriers, shifting the pulses and rotation is required. $\mathrm{M}$ sub symbols each having pulse, $\mathrm{M}$ sub-symbols each modulated on $\mathrm{K}$ subcarriers summed together to get total symbol, if the value of $M$ is reduced, bandwidth is same. This scenario is considered in three cases such as 1 ) $M=1, K$ increases to become $\mathrm{N}$. (no of subcarriers goes to $\mathrm{N}$, bandwidth is same) then the spacing between the subcarriers is present, GFDM becomes OFDM. 2)when $\mathrm{K}=0$, then $\mathrm{M}=\mathrm{N}$, it becomes SC-modulation, (no orthogonality between the subcarriers) but all $\mathrm{M}$ sub symbols in a series all these $\mathrm{M}$ have to modulated on a single pulse , this leads to single carrier FDE(SC-FDE). 3) By varying the values of M\&T, the bandwidth associated with these pulses can be varied, when $M=1$. Subcarrier spacing is $1 / T$ which is very small value, if the parameter $\mathrm{M}$ increases the bandwidth is increased. For some applications, the subcarriers need more bandwidth, in GFDM, for a combination of $\mathrm{M}$ sub-symbols and $\mathrm{K}$ subcarriers all combine together to get one guard interval. IFFT or IDFT can be used in transmitter side.

\subsection{GFDM Transmitter using LABVIEW}

The Galois PN Sequence generator generates the binary digits (bits) randomly. It is applied to the convolutional encoder VI and followed by hamming encoder VI and this encoded sequence is applied to GFDM modulator circuit which generates filter coefficients depend on different pulse shaping filters such as RRC, RC and Gaussian. FFT VI computes the fast Fourier transform for the input sequence, 16 size constellation QAM is used for GFDM modulation. The complex values of GFDM is are displayed in front panel, the data is stored in IQ data Queue.

$$
g_{k, m}[n]=g[(n-m k) \bmod N] \cdot e^{\left[-j 2 \pi \frac{k}{K} n\right]}
$$

Subcarrier-wise digital pulse shaping and subcarrier up conversion is applied.Each $d_{k, m}$ is transmitted with the corresponding pulse form. Each $\mathrm{g}_{\mathrm{k}, \mathrm{m}}[\mathrm{n}]$ may be a time and frequency shifted version of a imagefilter $\mathrm{g}[\mathrm{n}]$. The modulo operation makes $g_{k, m}[n]$ a circularly shifted version of $g_{k, 0}[n]$ The complicated exponential performs the shifting operation in frequency. The transmit image $[n]$ obtained by superposition of all transmit symbol.

$$
\begin{aligned}
& x[n]=\sum_{k=0}^{K-1} \sum_{m=0}^{M-1} g_{k, m}[n] d_{k, m} \\
& \overrightarrow{\boldsymbol{x}}=\mathbf{G} \overrightarrow{\boldsymbol{d}}
\end{aligned}
$$

\subsection{GFDM Receiver using LABVIEW}

Time and frequency synchronization are performed, yielding $\mathrm{y}_{\mathrm{s}}$. The cyclic prefix is removed by using subVI. The Van de Beek algorithm is used to decode the input sequence i.e., maximum likelihood detection. The signal is passed through channel estimation subVI which produces equalized data as shown in the front panel.

$$
\begin{aligned}
& \vec{y}=\vec{H} \vec{x}+\vec{z} \\
& B_{\text {Zeroforcing }}=G^{-1} \\
& \bar{d}=B \vec{y}
\end{aligned}
$$




\subsection{Convolutional Codes}

They are the second class of error correcting codes. Initially they are used in spatial transceivers. The sequence of data bits enters into the encoder one at a time, and it generates $n$ parity coded binary digits are transmitted to the channel, the ML decoder is used in the LABVIEW receiver program for decoding of bits. Figure 3 shows the working operation of convolutional encoder.

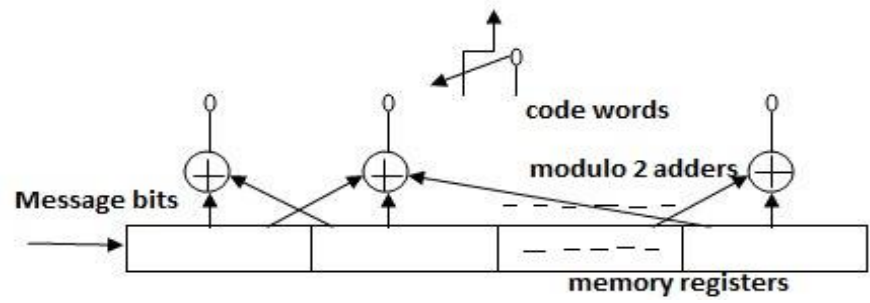

Figure 3. Convolutional encoder

Depending on the linear operation the encoder generates the bits, with the help of code rate $\mathrm{k}=\mathrm{r} / \mathrm{n}$ and shift register update the number of bits are processed, i.e. (n,k,K) and measures the efficiency[18]. n represents the length of the code word, $\mathrm{k}$ represents number of input data bits, $\mathrm{K}$ represents constraint length. The Figure 4 shows the dimension of convolutional code (7, [171 133]) is display.with a constraint length of 7, generator polynomials G1 = 171 and G2 $=133$. The feedback connections are [133 octal] and [171 octal] with generator upper polynomial matrix, [ $\left.\begin{array}{lll}1 & 0 & 1\end{array}\right]$ and under polynomial matrix, [lll 11 1 [ 19$]$. The traditional algorithm of convolutional encoder is maximum likelihood estimation algorithm-ML algorithm (Van de Beek et al., 1997) which has low computational complexity, which is used as decoder for this proposed system as subVI in LABVIEW program.

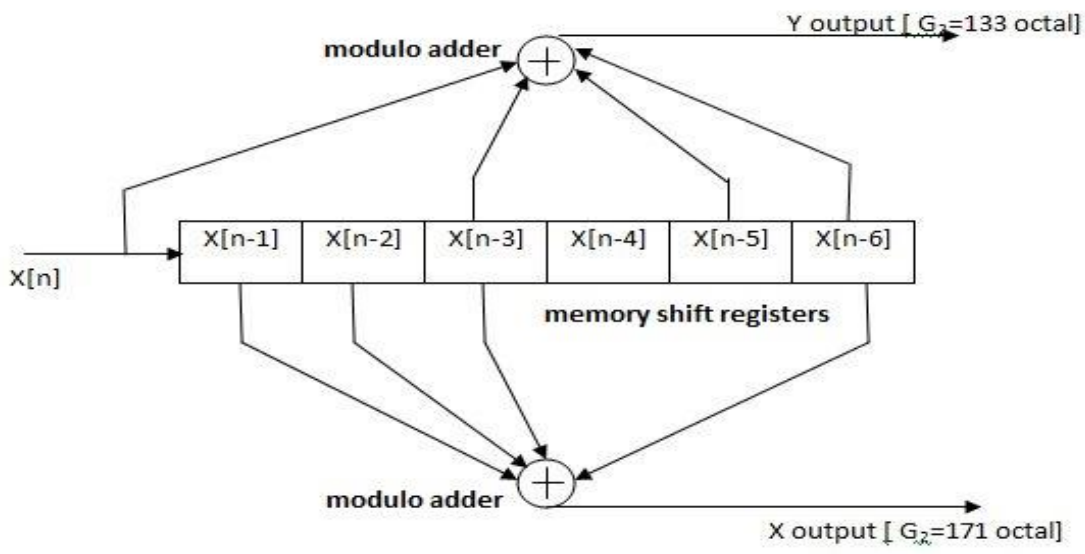

Figure 4. 7,[171 133] Convolutional encoder with $\mathrm{K}=7$

\subsection{Hamming Encoder}

The Hamming codes are also one of linear block codes, which detect and correct binary digits [15]. Figure 5 shows the entire block of hamming code which has parity bits along with message bits. k represents the message bits and q represents parity bits. 


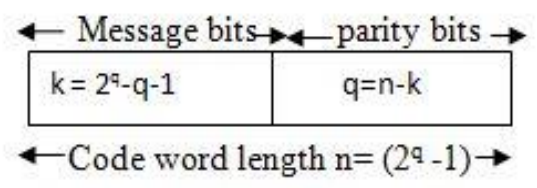

Figure 5. Hamming code word with message and check bits

When the minimum distance i.e, $d_{\min }=3$ the code is said to be hamming code. The detection of two or more errors within the block, Hamming code is one of the commonest codes used in the protection of information from error The error detection and correction capabilities of a coding technique depend on the minimum distance $\mathrm{d}_{\min }[20]$.

$\mathrm{d}_{\min } \geq(\mathrm{j}+1)$

$\mathrm{d}_{\min } \geq(2 \mathrm{z}+1)$

$\mathrm{d}_{\min } \geq(\mathrm{j}+\mathrm{z}+1)$

The encoder takes $\mathrm{n}$ bits input data and produces a $\mathrm{k}$ bit codeword. The encoder was designed through the usual generator matrix multiplication while in the decoder design the computation of the syndrome vector was ignored. Meanwhile, the different states that can represent a particular input were calculated and the decoder was designed to identify each codeword representing a particular input. Results have shown that the method is also reliable, equation (7) can detect errors up to $\mathrm{j}$ errors per word, equation (8) can correct errors up to $\mathrm{z}$ errors per word. Equation (9) can correct up to $\mathrm{z}$ errors and detect $\mathrm{j}>\mathrm{z}$ errors per word.

\section{ALGORITHM FOR DESIGNING VIRTUAL INSTRUMENTATION TRANSCEIVER 3.1 GFDM Transmitter VI program}

Figure 6 shows the main VI Program of GFDM transmitter in which the input parameters such as IQ gain imbalance in decibels, the roll-off factor and filter defining function such as RRC and RC can be taken as user defined. The constellation size of QAM can also be defined according to the user defined specifications. The generated GFDM complex symbols are shown in front panel of the LABVIEW. The time domain GFDM signal is generated, and it is plotted with the graph. The pink colour datatype input is IQ Data Queue which stores the values from executed program. The programming structure was taken from the reference [21]. The OFDM is integrated with MIMO using USRP devices in virtual and remote lab. The signal processing tools using RF communications module was used [22]. The transceiver with different flow chart for transmitter and receiver separately was explained. The simulation of basic analog modulation schemes was taken from [23].

Figure 7 shows the subVI program of the GFDM VI transmitter. This program has step by step sequence of data flow from input from output. Firstly the filter coefficients are generated according to roll off factor value, and then coefficients are connected to array subset which generates sub-array for the length of $\mathrm{N}$ sequence. The Sequence is connected to 1D Rotate array which rotates the number of elements indicated by the length $\mathrm{n}$, then the sequence is connected to FFT VI, which generates frequency domain sequence, this sequence is splitted, i.e. series to parallel converter, this parallel sequences values are applied to for loop, inside the for loop the two stages of encoders are applied to the PN Sequence along with 16-QAM constellation size along with 256 subcarriers size of IFFT, the GFDM signal is generated with two dimensional format with $\mathrm{M}$ sub-symbols and $\mathrm{K}$ subcarriers in complex format. The signal is shown in time domain is plotted with the help of IFFT VI.

Table 1 shows the simulation parameters used in the transmitte VI, in which the programming used different roll-off factor values, along with subsymbols and subcarriers, the total symbols generated in the program is 1280 . 


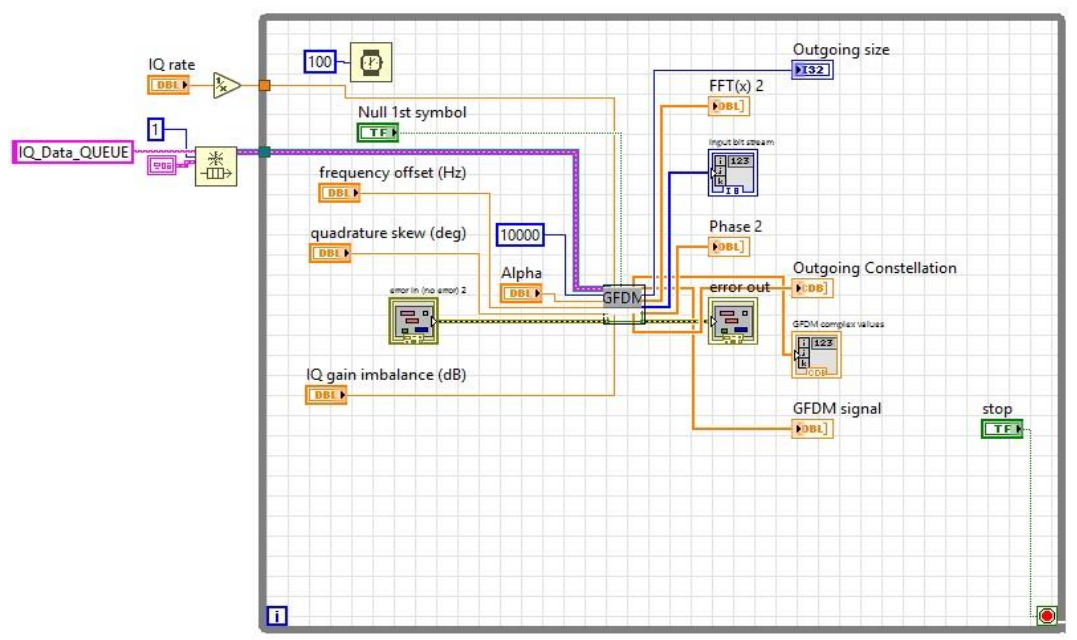

Figure 6. Main Program of Channel coded GFDM Transmitter VI

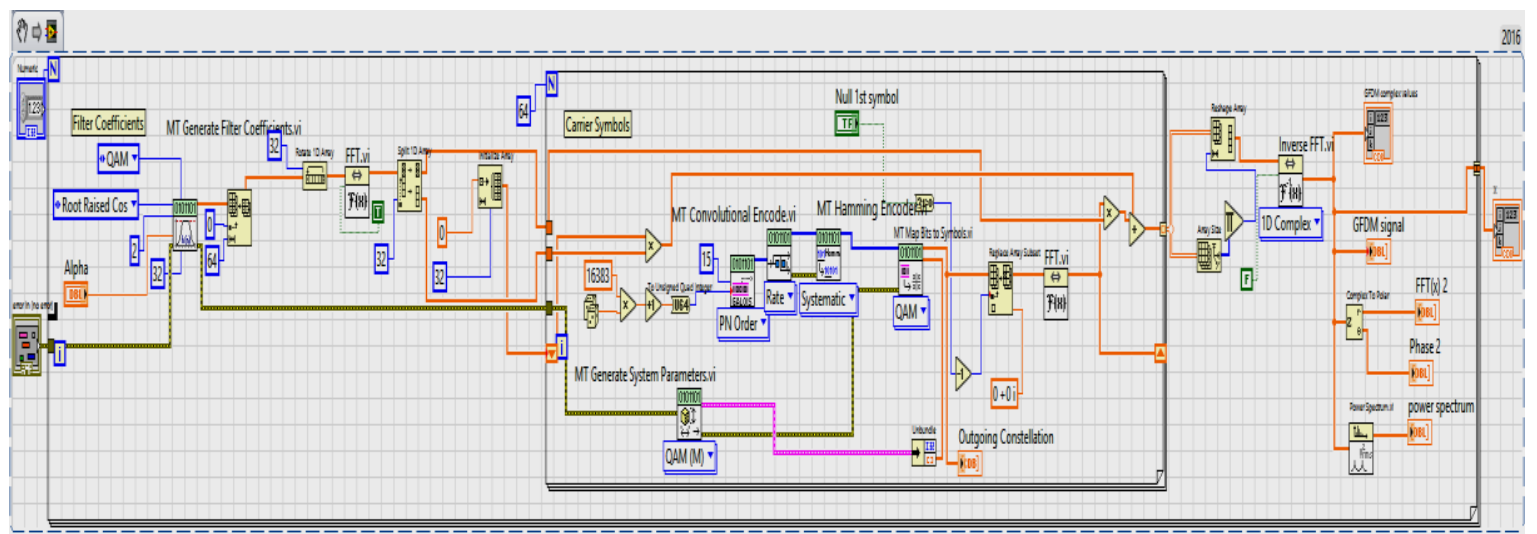

Figure 7. Sub VI of Channel coded GFDM Transmitter

Table 1. Simulation parameters used in transmitter VI

\begin{tabular}{cc}
\hline Parameter at Transmitter & Values used \\
\hline Alpha roll off factor (pulse shaping filter) & $0.3,0.5$ \\
IQ Rate & $5 \mathrm{M}$ \\
Frequency offset & 100 \\
Number of Samples & 150000 \\
IQ gain imbalance & 5 \\
Constellation size & 16 \\
Sub symbols M & 5 \\
subcarriers K & 256 \\
An Exponent of power delay profile & 10 \\
No of symbols & $\mathrm{N}=\mathrm{K} * \mathrm{M}=1280$ \\
\hline
\end{tabular}

\subsection{Receiver VI Program}

Figure 8 and Table 2 shows the receiver VI program and its simulation parameters used. The IQ Data Queue stores the output bits of transmitter VI, the IQ data is applied to van de Beek algorithm for maximum likelihood detection of complex values. The decoding of the NOMA signal is done using the subVI. The demodulation of GFDM signal is done by using the subVI. The cyclic prefix of the signal is removed. FFT is used to convert the frequency domain signal to time domain signal. The zero padding subVI is used to remove all the zeros added at the transmitter. The channel estimation algorithm is used to make unequalizsed data to equalized data as shown in graph. Then the series levels of two concenated codes are used at the decoder. After the decoding process, the corresponding BER is calculated between the input bit stream and output bit stream. For different values of SNR, the corresponding BER is plotted. 


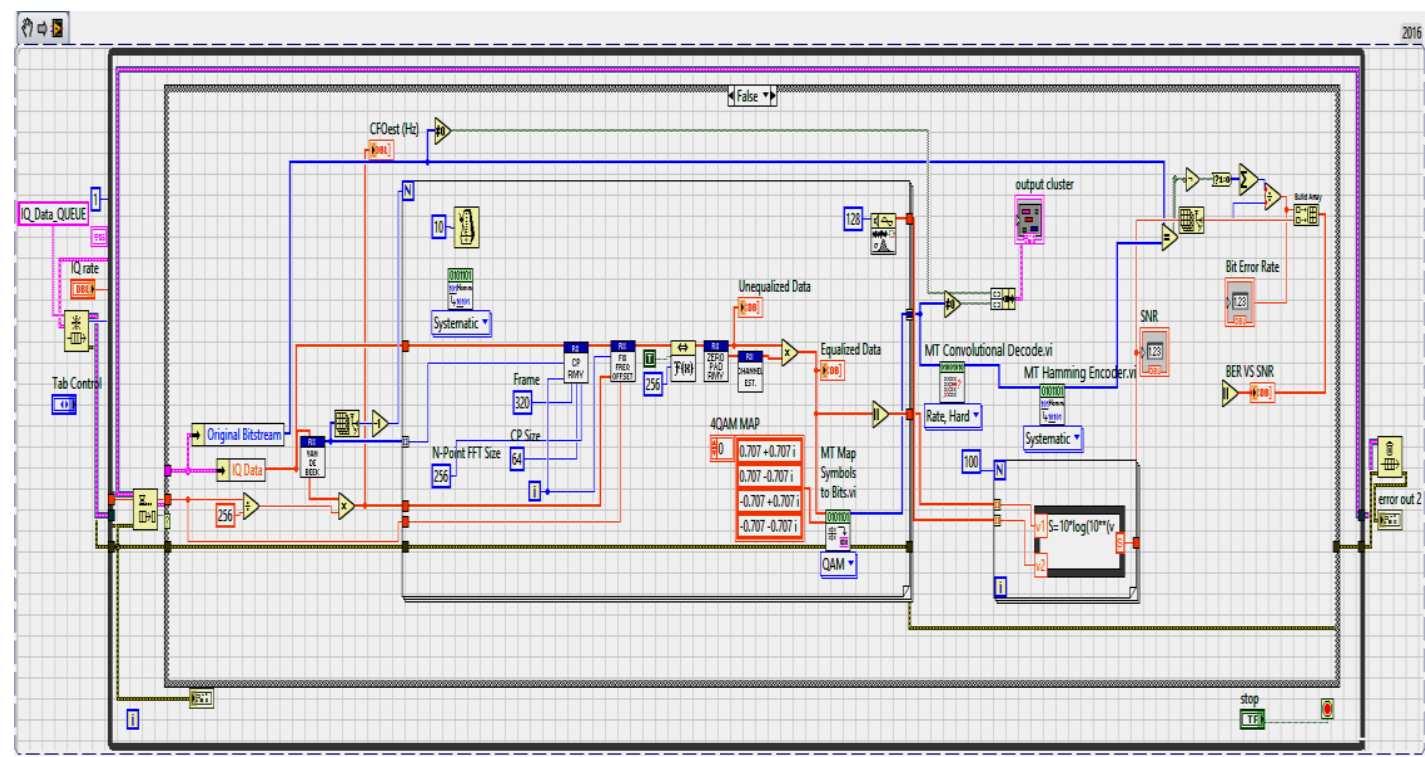

Figure 8. Channel coded GFDM Receiver VI program

Table 2. Simulation parameters used in Receiver VI

\begin{tabular}{cc}
\hline Parameter at Receiver & Values used \\
\hline IQ Rate & $5 \mathrm{M}$ \\
Number of Samples & 150000 \\
Delay spread $\mu \mathrm{s}$ & 5 \\
$B_{c} \mathrm{KHz}$ & 5 \\
Coherence bandwidth ${ }^{\mathrm{K}}$ & 200 \\
Doppler shift $\mathrm{Hz}$ & 100 \\
$T_{c} \mathrm{~ms}$ & 10 \\
Coherence time & 2 \\
Cyclic prefix time $\mu \mathrm{s}$ & 5 \\
Symbol duration $\mu \mathrm{s}$ & 100 \\
Bandwidth $\mathrm{KHz}$ & \\
SNR & {$[1-10] \mathrm{dB}$} \\
\hline
\end{tabular}

\section{RESULTS AND DISCUSSIONS}

GFDM complex values are generated as shown in screenshot. They are represented using one dimensional array format. The characteristics of GFDM signal and its frequency domain and phase angle is plotted with graph. The roll-off factor value is 0.9 for corresponding pulse shaping filter.Figure 9 and Table 1 shows the simulation parameters used, and transmitter VI front panel shows different graphs of each section. GFDM complex values and input bit streams are shown, with roll off factor value. The power spectrum of signal is plotted with the graph, and 16 constellation points are shown. The removal of cyclic prefix and zero padding is done by inbuilt subVI and complex values of GFDM signal is applied to channel estimation, the equalized data is plotted at the front panel as shown in Figure 10. The convolutional decoder and hamming decoder are used before the constellation demapping of 16-QAM. Finally the output bit stream is generated as shown in Figure 10 with LED. The generated output bit stream and the corresponding input bit stream is shown in front panel. The BER VI is available in the LABVIEW, by using it the BER value is calculated. The data is normalized for the plot using scaling function. The BER values are plotted for different values of SNR in decibels.

Table 3 shows the simulation results of BER for SNR values of $5 \mathrm{~dB}$ and $9 \mathrm{~dB}$. The BER value is calculated for one stage level of channel coding with two algorithms, then followed by two stage levels of encoding and decoding the BER is improved by $45.91 \%$ when compared to single stage encoder stage. 


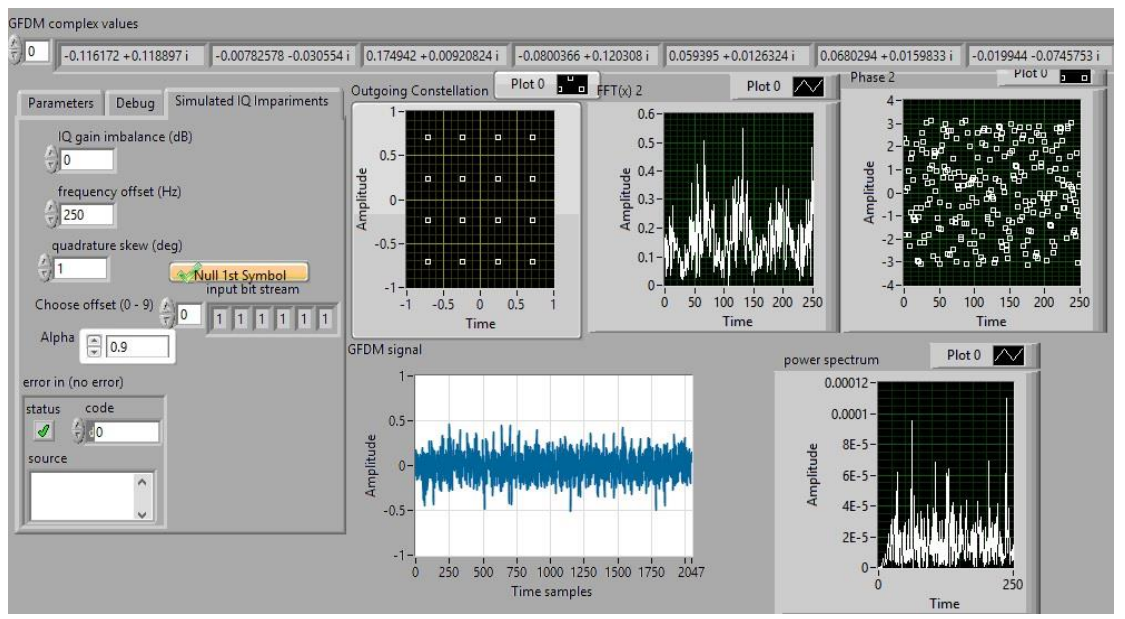

Figure 9. Front panel screenshot of Channel coded GFDM transmitter VI program

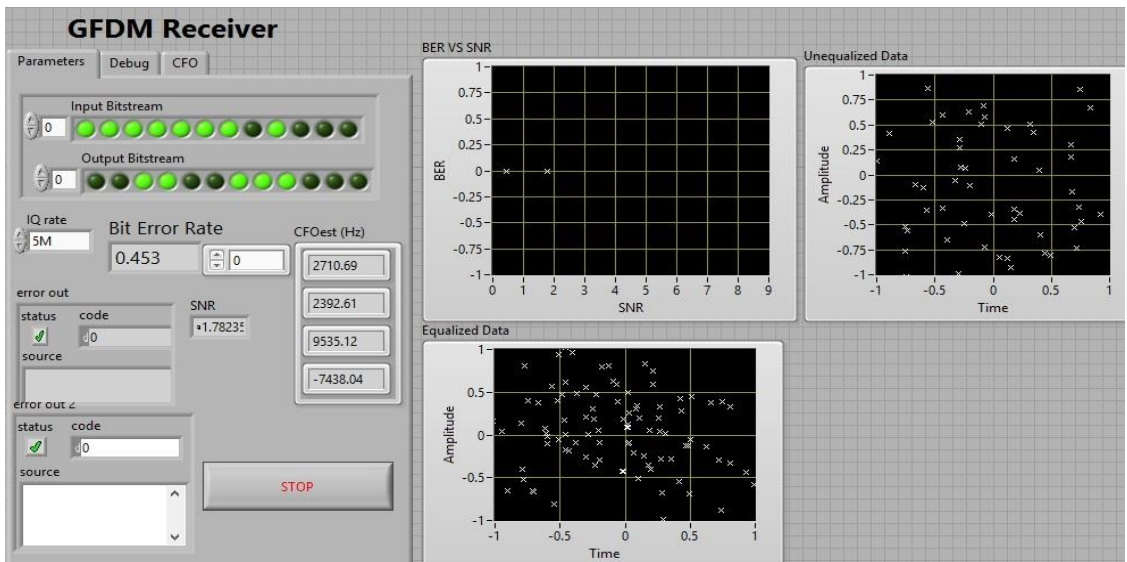

Figure 10. Front panel screenshot of Channel coded GFDM Receiver VI program

Table 3. BER Analysis in channel coded Receiver VI

\begin{tabular}{ccc}
\hline Channel Coding algorithm & BER at SNR 5 dB & BER at SNR 9 dB \\
\hline Only convolutional coding & $7.851 \times 10^{-5}$ & $6.85 \times 10^{-5}$ \\
Only hamming coding & $6.6414 \times 10^{-4}$ & $5.587 \times 10^{-4}$ \\
Two hamming coding & 0.000254 & 0.0001215 \\
Two convolutional coding & $5.254125 \times 10^{-4}$ & $3.9854 \times 10^{-4}$ \\
Hamming and convolutional & $2.58 \times 10^{-5}$ & $3.45 \times 10^{-6}$ \\
series codes & $4.7512 \times 10^{-5}$ & $3.874 \times 10^{-5}$ \\
Without channel coding & &
\end{tabular}

Figure 11 shows the simulation result of fixed modulation scheme and without channel coding employed. This program is run using RT MATHSCRIPT module in LABVIEW which simultaneously run both MATLAB and LABVIEW software. The SER is compared with the proposed system, the channel coded GFDM system provides less BER compared to the existing system. 


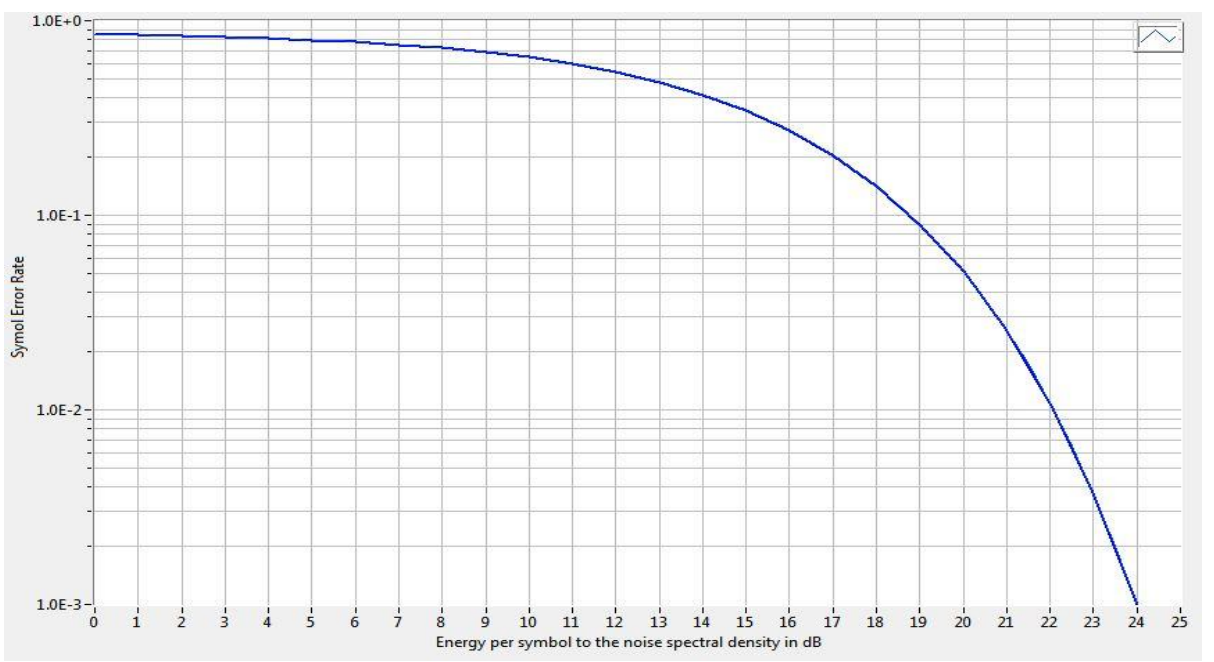

Figure 11. SER for GFDM without channel coding using RT MATHSCRIPT MODULE

\section{CONCLUSION}

This paper gives the BER performance of the GFDM system with concatenated levels of channel encoded algorithms. Channel encoder utilized in the correspondence framework to identify and redress the binary digits errors because of the channel impedances at the recipient. Picking the encoder/decoder composes that identify and right huge quantities of bits improve the execution of the correspondence framework. The coding calculation utilized in this paper is arrangement connected hamming/convolutional encoder/decoder levels. This calculation improves the framework execution by lessening BER at a reasonable SNR. The proposed system is compared with existing system of SER without channel encoding algorithms. The Bit error rate is calculated for 16-QAM, and 256 subcarriers, 5 sub-symbols for randomly generated PN Sequence with employing concatenated two stage levels of encoders at the transmitter VI. On the basis of the results procured in the present LABVIEW based simulation, we find that the proposed system provides a less bit error rate by applying two-stage encoders and decoders at the program. The proposed system achieves $32.91 \%$ improvement in the BER. This system outperforms the OFDM signal interms of BER.

\section{REFERENCES}

[1] Zeng Y, Liang YC, Chia MW, Pham TH. Fast Algorithms for FBMC and GFDM in Dynamic Spectrum Access. In2017 IEEE Wireless Communications and Networking Conference (WCNC) 2017 Mar 19 (pp. 1-6). IEEE.

[2] Yenilmez A, Gucluoglu T, Remlein P. Performance of GFDM-maximal ratio transmission over Nakagami-m fading channels. InWireless Communication Systems (ISWCS), 2016 International Symposium on 2016 Sep 20 (pp. 523-527). IEEE.

[3] Ortega A, Fabbri L, Tralli V. Performance evaluation of GFDM over nonlinear channel. InInformation and Communication Technology Convergence (ICTC), 2016 International Conference on 2016 Oct 19 (pp. 12-17). IEEE.

[4] Michailow N, Matthé M, Gaspar IS, Caldevilla AN, Mendes LL, Festag A, Fettweis G. Generalized frequency division multiplexing for 5th generation cellular networks. IEEE Transactions on Communications. 2014 Sep;62(9):3045-61.

[5] Gaspar D, Mendes L, Pimenta T. GFDM BER under synchronization errors. IEEE Communications Letters. 2017 Aug;21(8):1743-6.

[6] Wang PS, Lin DW. Maximum-likelihood blind synchronization for GFDM systems. IEEE Signal Processing Letters. 2016 Jun;23(6):790-4.

[7] Akai Y, Enjoji Y, Sanada Y, Kimura R, Sawai R. GFDM with different subcarrier bandwidths. InVehicular Technology Conference (VTC-Fall), 2016 IEEE 84th 2016 Sep 18 (pp. 1-5). IEEE.

[8] Michailow N, Mendes L, Matthé M, Gaspar I, Festag A, Fettweis G. Robust WHT-GFDM for the next generation of wireless networks. IEEE Communications Letters. 2015 Jan;19(1):106-9.

[9] Damnjanovic A, Montojo J, Wei Y, Ji T, Luo T, Vajapeyam M, Yoo T, Song O, Malladi D. A survey on 3GPP heterogeneous networks. IEEE Wireless communications. 2011 Jun;18(3).

[10] Khalili F. Design and Simulation of Coded-Modulation Using Turbo Trellis Coding and Multi-Layer Modulations (Doctoral dissertation, Ohio University). 
[11] Bawa M, Tahernezhadi M. Performance evaluation of convolutional encoded partial differential space time OFDM using modified local splines. InElectro/Information Technology (EIT), 2015 IEEE International Conference on 2015 May 21 (pp. 001-005). IEEE.

[12] Parvez AS, Rahman MM, Podder P, Hossain M, Islam MA. Design and Implementation of Hamming Encoder and Decoder Over FPGA. InInternational Conference on Computer Networks and Communication Technologies 2019 (pp. 1005-1022). Springer, Singapore.

[13] Alimi, Isiaka Ajewale, Kayode Francis Akingbad, Jide Julius Popoolae, and Michael O. Kolawole. An Hybrid Coding Technique for Efficient Bandwidth Usage in conformity with IEEE 802.11 WLAN Standard. International Journal of Electrical and Computer Engineering (IJECE), 2013, 3(5): 593-602.

[14] Oluwafemi, Ilesanmi Banjo. "HybridConcatenated Coding Scheme for MIMO Systems. International Journal of Electrical and Computer Engineering (IJECE),2015 5(3): 464-476.

[15] Lodro, Mir Muhammad, and Muhammad Hanif Abro. Ergodic Capacity of MIMO Correlated Channels in Multipath Fading Environment with known Channel State Information. International Journal of Electrical and Computer Engineering (IJECE),2012. 2(5): 691-697.

[16] Hendre, Vaibhav S., M. Murugan, and Sneha Kamthe. Performance Analysis of Transmit Antenna Selection with MRC in MIMO for Image Transmission in Multipath Fading Channels Using Simulink. International Journal of Electrical and Computer Engineering (IJECE), 2015, 5(1): 119-128.

[17] Ali, Noura, M. Ibrahim Youssef, and I. Fathi Tarrad. Performance Improvement by using a Concatenated Levels of Encoding in Wavelet based OFDM Systems. International journal of computer applications, 2017, 177(5):30-34.

[18] Sklar, B., (2001). Digital communications (Vol. 2). Upper Saddle River: Prentice Hall.

[19] John.GProakis (2008), Digital communications, Fifth edition,

[20] Sharma, Sanjay (2012), Communication Systems: Analog and Digital. SK Kataria and sons.

[21] Telagam N, Kandasamy N, Nanjundan M. Smart Sensor Network Based High Quality Air Pollution Monitoring System Using Labview. International Journal of Online Engineering (iJOE), 2017 Aug 4;13(08):79-87.

[22] Somanaidu, Utlapalli, Nagarjuna Telagam, Nehru Kandasamy, and Menakadevi Nanjundan. USRP 2901 Based FM Transceiver with Large File Capabilities in Virtual and Remote Laboratory. International Journal of Online Engineering (iJOE) 2018, oct, 14(10) : 193-200.

[23] Kandasamy N, Telagam N, VR SR, Arulananth TS. Simulation of Analog Modulation and Demodulation Techniques in Virtual Instrumentation and Remote Lab. International Journal of Online Engineering (iJOE). 2017 Nov 7;13(10):140-7

\section{BIOGRAPHIES OF AUTHORS}

Mr. T.Nagarjuna is a research scholar in the school of electronics in sathyabama (deemed to be
university). He can be reached at nagarjuna473@ gmail.com

